\title{
The GRACE project - QCD, SUSY, Multi-Ioop -
}

\section{J. Fujimoto*, N. Hamaguchi, T. Ishikawa, T. Kaneko, Y. Kurihara, S. Odaka, Y. Shimizu, F. Yuasa,}

High Energy Accelerator Research Organization (KEK), 1-1 Oho Tsukuba, Ibaraki 305-0801, Japan

E-mail: junpei.fujimoto@kek.jp, hamagu@post.kek.jp,

tadashi.ishikawa@kek.jp, toshiaki.kaneko@kek.jp,

shigeru.odaka@kek.jp, yoshimasa.kurihara@kek.jp, shimiz@suchix.jp,

fukuko.yuasa@kek.jp

\section{T. Inoue, T. Koike, T. Kon,}

Seikei University, Musashino, Tokyo 180-8633, Japan

E-mail: dm106140cc@cc.seikei.ac.jp, dm106119@cc.seikei.ac.jp,

konest.seikei.ac.jp

\section{Jimbo}

Chiba University of Commerce, Ichikawa, Chiba 272-8512, Japan

E-mail: jimbo@cuc.ac.jp

\section{K. Kato}

Kogakuin University, 1-24 Nishi-Shinjuku, Shinjuku, Tokyo 163-8677, Japan

E-mail: kato@cc.kogakuin.ac.jp

\section{Kuroda}

Meiji Gakuin University, Yokohama, Kanagawa 244-8539, Japan

E-mail: kurodamelaw.meijigakuin.ac.jp

\section{E. de Doncker}

Western Michigan University, Kalamazoo, MI 49008-5371, USA

E-mail: elise.dedoncker@wmich.edu

Recent studies of QCD and MSSM processes are discussed. These processes are handled with the GRACE system, which is a package of the automatic calculation of the amplitude based on the standard model (SM) and the minimal supersymmetric extension of the standard model (MSSM), are discussed. In order to evaluate multi-loop diagrams, we have developed the direct computation method (DCM). As an application example, two-loop non-planar box diagrams are evaluated by method.

10th International Symposium on Radiative Corrections (Applications of Quantum Field Theory to Phenomenology) - Radcor 2011

September 26-30, 2011

Mamallapuram, India

\footnotetext{
* Speaker.
} 


\section{Introduction}

High energy physics at the current and future colliders like the LHC and the ILC requires theorists to carry out large-scale calculations with multi-body productions. In the perturbative approach, the number of Feynman graphs to be calculated grows rapidly as the number of produced particles increases and as the order of perturbation becomes higher. For example, several tens of diagrams typically appear in the final four-body processes at the tree level, but a few thousand diagrams emerge in the 1-loop correction. Performing such computation is absolutely beyond the human power if it should be done by hand. Since the procedure of a perturbation calculation is well established, computers must be able to take the place of the human hand. In the past decade, several groups have developed computer programs which generate Feynman diagrams and calculate cross sections automatically. The GRACE system is such a program package for the automatic calculation of the amplitudes, based on the Feynman rules of the standard model (SM) and also of the minimal supersymmetric extension of the standard model (MSSM). We report the current status of studies with the GRACE system on QCD and MSSM. Furthermore we also take the two-loop non-planar box diagram as an example to treat loop-diagrams beyond one-loop diagrams.

\section{QCD application for the LHC}

A program package of GR@PPA (GRace At Proton-Proton/Antiproton) version 2.8 was released in November, 2010 [1] $]^{1}$. GR@PPA is an extension of the GRACE system [2, 3] to hadron collision interactions. GRACE is a powerful tool for deriving the differential cross section of hard interactions at the parton level, and for generating the corresponding events with the help of BASES/SPRING [4, 5]. GR @PPA provides a mechanism for adding the effects of the initial-state variation in the flavor and momentum according to the parton distribution finctions (PDF), and for achieving a generalization of the final state. The previous releases of GR@PPA [6, 7] included many multi-body (multi-jet) production processes, such as $W+$ jets and $Z+$ jets, diboson $\left(W^{+} W^{-}\right.$, $W Z, Z Z)+$ jets, top-pair + jets, and QCD multi-jets. Although the version 2.8 includes only those processes for single and double weak-boson productions associated with the jet production up to one jet, a jet matching method is applied to simulate the weak-boson kinematics continuously in the entire phase space.

In order to accomplish an appropriate jet matching, we subtract divergent collinear components numerically from radiative (1 jet) processes. The subtracted components are identical to the leading terms in parton showere (PS), applied to non-radiative $(0$ jet) processes. Thus, we can obtain smooth spectra by combining the subtracted 1 -jet processes with the 0 -jet processes to which an appropriate PS is applied, with allowing any divergence or double-counting problem. Since the PS implementation is limited with a certain energy scale $\left(\mu_{P S}\right)$, the subtraction is limited with the same scale. In addition, we take $\mu_{P S}$ equal to the factorization scale in order to strictly preserve the identity between the PS and PDF.

We have shown in a previous report [8] that the simulation employing our matching method and a custom-made initial-state parton shower reproduces the $p_{T}$ spectrum of the $Z$ bosons measured at Tevatron experiments $[9,10,11]$, with very good precision. Figure 1 shows the result

\footnotetext{
${ }^{1}$ http://atlas.kek.jp/physics/nlo-wg/grappa.html.
} 

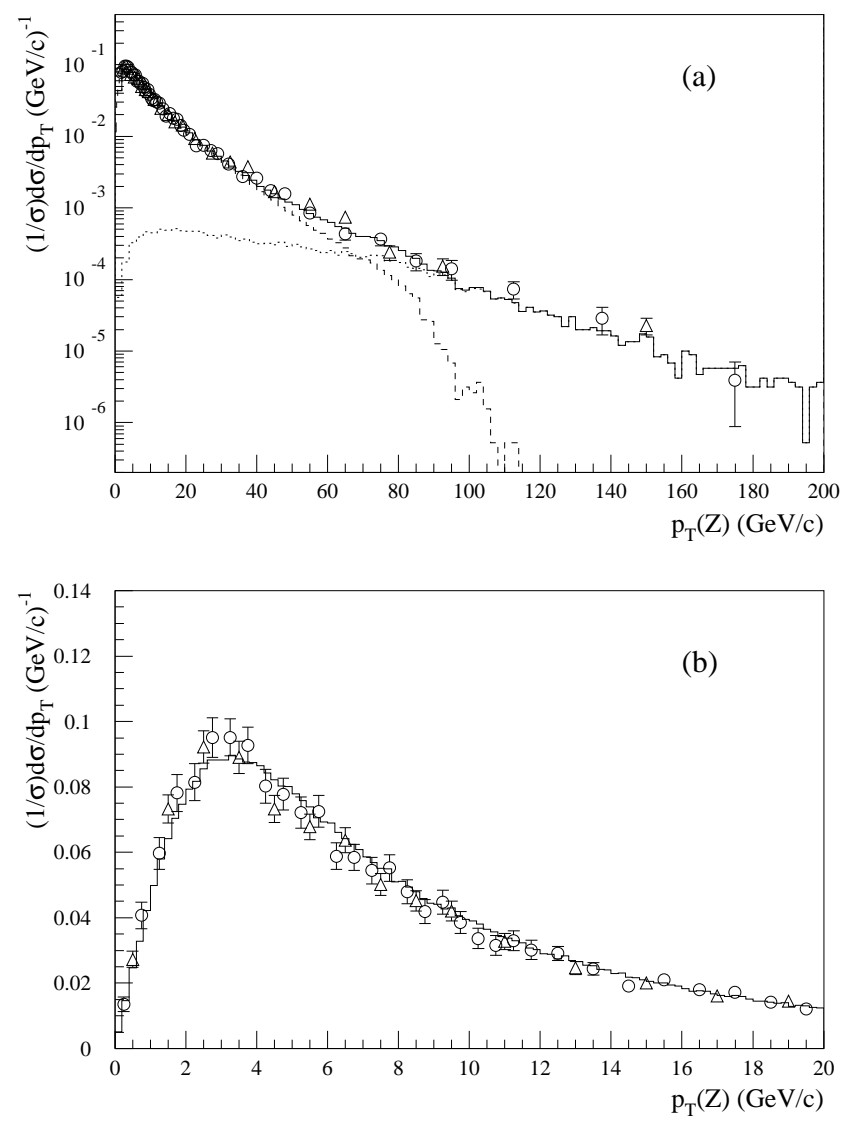

Figure 1: $\quad p_{T}$ spectrum of $Z$ bosons at Tevatron Run 1, p $\bar{p}$ collisions at a cm energy of $1.8 \mathrm{TeV}$. The GR@PPA simulation (histograms) is compared with the measurements by CDF [9] (circles) and D0 [10] (triangles). Together with a result covering the $p_{T}$ range up to $200 \mathrm{GeV} / c$ (a), a result to cover the range up to $20 \mathrm{GeV} / c$ (b) is presented to show the low- $p_{T}$ behavior. In addition to the summed spectrum (solid line), the spectra of events from the $Z+0$-jet (dashed line) and $Z+1$-jet (dotted line) processes are shown separately for the GR@PPA simulation in (a).

of a simulation of the same quantity, in which, together with the initial-state PS, a final-state PS is applied to partons from the hard interaction and to those radiated in the initial-state PS. The factorization scale $\left(\mu_{F}\right)$ and the renormalization scale $\left(\mu_{R}\right)$ are taken to be equal to the $Z$-boson mass $\left(91.17 \mathrm{GeV} / c^{2}\right)$. We can see that the $Z+0$-jet component overwhelms the remaining non$\operatorname{logarithmic} Z+1$-jet component for low $p_{T}<\sim 100 \mathrm{GeV} / c$, and the non-logarithmic component converges to zero as $p_{T} \rightarrow 0$. The summed spectrum is somewhat softer compared to the previous result. Nevertheless, because of the application of the final-state PS, the simulation is still in good agreement with the measurements.

\section{MSSM application}

Despite its compactness and success in describing known experimental data available up to 
now, the standard model (SM) is considered to be an effective theory which is valid only at the currently accessible energies, on account of theoretical problems. Supersymmetric (SUSY) theory, which predicts the existence of a partner to every particle of the SM that differs in spin by one half, is believed to be an attractive candidate for the theory beyond the SM (BSM). The minimal supersymmetric extension of the SM (MSSM) remains consistent with all known high-precision experiments at a level comparable to the SM. One of the most important aims of the particle experiments at sub-TeV-region and TeV-region energies is to probe evidence of the BSM; so the search for SUSY particles plays a crucial role in it.

Experiments at present and future accelerators, the Large Hadron Collider (LHC) and the International Linear Collider (ILC), are expected to discover SUSY particles and provide accurate data on them. In particular, experiments at the ILC offer high-precision determination of SUSY parameters via $e^{-} e^{+}$-annihilation processes. Since theoretical predictions with similarly high accuracy are required for us to extract important physical results from the data, we have to include at least one-loop contributions in perturbative calculations of the amplitude.

Among SUSY particles, only the lightest one (LSP) is stable if $R$-parity is conserved. Then, decay processes should be analyzed precisely in experiments at the LHC and the ILC. Recently, we have calculated the radiative corrections to production processes and decay processes of SUSY particles, in the framework of the MSSM using GRACE/SUSY-loop [12, 13, 14, 15]. Related references can be found in [16].

For many-body final states, each production process or decay process is described by a large number of Feynman diagrams, even at tree-level order. There are still more Feynman diagrams at one-loop order even for two-body final states. For this reason, we have developed the GRACE system [3], which enables us to calculate amplitudes automatically. A program package called GRACE/SUSY-loop is a version of the GRACE system for the calculation of the MSSM amplitudes in one-loop order, which includes the model files of the MSSM and the loop library. There exist other program packages developed independently by other groups, for the calculation of the MSSM amplitudes at one-loop order, SloopS [17] and FeynArt/Calc [18].

We have calculated the radiative corrections to production processes and decay processes of SUSY particles in the framework of the MSSM using GRACE/SUSY-loop. Table 1 shows the list of processes calculated using GRACE/SUSY-loop.

\begin{tabular}{lcc}
\hline Processes & GRACE & Preceding studies \\
\hline Chargino-pair production $\left(e^{-}+e^{+} \rightarrow \widetilde{\chi}_{1}^{-}+\widetilde{\chi}_{1}^{+}\right)$ & {$[12]$} & {$[19,20,21]$} \\
Chargino decay $\left(\widetilde{\chi}_{2}^{+} \rightarrow\right.$ two body and $\widetilde{\chi}_{1}^{+} \rightarrow$ three body $)$ & {$[12]$} & \\
Neutralino-pair production $\left(e^{-}+e^{+} \rightarrow \widetilde{\chi}_{1}^{0}+\widetilde{\chi}_{2}^{0}\right)$ & {$[13]$} & {$[19,20]$} \\
Neutralino decay $\left(\widetilde{\chi}_{2,3,4}^{0} \rightarrow\right.$ two body and $\widetilde{\chi}_{2}^{0} \rightarrow$ three body $)$ & {$[13]$} & {$[22]$} \\
Sfermion decay $(\widetilde{f} \rightarrow$ two body $)$ & {$[14]$} & {$[23,24,25]$} \\
Stop production $\left(e^{-}+e^{+} \rightarrow \widetilde{t}_{1}+\widetilde{t}_{1}^{*}\right)$ & {$[26]$} & {$[27]$} \\
Stop decay $\left(\widetilde{t}_{1} \rightarrow b+\widetilde{\chi}_{1}^{+}, t+\widetilde{\chi}_{1}^{0}\right.$ and $\left.\widetilde{t}_{1} \rightarrow b+W^{+}+\widetilde{\chi}_{1}^{0}\right)$ & {$[15]$} & {$[23,24,25]$} \\
Gluino decay $\left(\widetilde{g} \rightarrow b+\widetilde{b}_{1}, t+\widetilde{t}_{1}\right)$ & {$[15]$} & {$[23]$} \\
\hline
\end{tabular}

Table 1: List of processes calculated using GRACE/SUSY-loop 


\section{Two-loop non-planar box diagrams}

At the one-loop level, analytic solutions exist for any diagrams. These are expressed in terms of known functions, such as logarithms and Spence functions (see, for example [28]).

The extension to calculate higher order corrections is, however, not a trivial task, because analytic expressions of the integrals are generally unknown for higher-loop diagrams, especially for diagrams with more general mass configurations. In order to overcome this situation, we rely on numerical evaluations. We need to establish efficient methods that can be incorporated into automatic computation systems to evaluate cross-sections. Over the past decade we have accumulated experience evaluating one-loop integrals numerically, since those results can be compared with analytic answers. We succeeded in calculating vertex, box and pentagon diagrams with arbitrary masses. We also computed two-loop self-energy and vertex diagrams. Related work and references can be found in [29].

In this paper we focus on the topology in Fig. 2, called the non-planar box diagram. The loop integral in the Feynman parameters $\left(x_{1}, \cdots, x_{7}\right)$ is expressed as follows;

$$
I=-\int_{0}^{1} d x_{1} d x_{2} d x_{3} d x_{4} d x_{5} d x_{6} d x_{7} \delta\left(1-\sum_{\ell=1}^{7} x_{\ell}\right) \frac{C}{(D-i \varepsilon C)^{3}} .
$$

Here, $D$ and $C$ are polynomials of Feynman parameters.

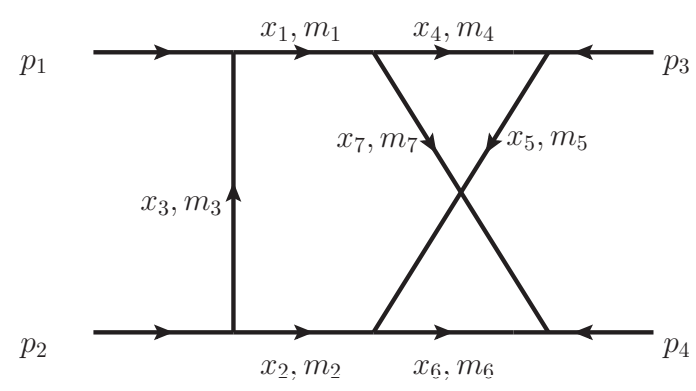

Figure 2: Two-loop non-planar box diagram

The external momenta $p_{1}, p_{2}, p_{3}$ and $p_{4}$ are defined to flow inward, satisfying $p_{1}+p_{2}+p_{3}+p_{4}=0$. The kinematical variables $s$ and $t$ are given by $s=\left(p_{1}+p_{2}\right)^{2}=\left(p_{3}+p_{4}\right)^{2}, t=\left(p_{1}+p_{3}\right)^{2}=\left(p_{2}+\right.$ $\left.p_{4}\right)^{2}$. For later notational convenience we introduce a third kinematical variable $u=\left(p_{1}+p_{4}\right)^{2}=$ $\left(p_{2}+p_{3}\right)^{2}$.

The functions $D$ and $C$ in Eq. (4.1), corresponding to Fig. 2, are

$$
\begin{aligned}
D & =C \sum x_{\ell} m_{\ell}^{2} \\
& -\left\{s\left(x_{1} x_{2} x_{4}+x_{1} x_{2} x_{5}+x_{1} x_{2} x_{6}+x_{1} x_{2} x_{7}+x_{1} x_{5} x_{6}+x_{2} x_{4} x_{7}-x_{3} x_{4} x_{6}\right)\right. \\
& +t\left(x_{3}\left(-x_{4} x_{6}+x_{5} x_{7}\right)\right) \\
& +p_{1}^{2}\left(x_{3}\left(x_{1} x_{4}+x_{1} x_{5}+x_{1} x_{6}+x_{1} x_{7}+x_{4} x_{6}+x_{4} x_{7}\right)\right) \\
& +p_{2}^{2}\left(x_{3}\left(x_{2} x_{4}+x_{2} x_{5}+x_{2} x_{6}+x_{2} x_{7}+x_{4} x_{6}+x_{5} x_{6}\right)\right) \\
& +p_{3}^{2}\left(x_{1} x_{4} x_{5}+x_{1} x_{5} x_{7}+x_{2} x_{4} x_{5}+x_{2} x_{4} x_{6}+x_{3} x_{4} x_{5}+x_{3} x_{4} x_{6}+x_{4} x_{5} x_{6}+x_{4} x_{5} x_{7}\right) \\
& \left.+p_{4}^{2}\left(x_{1} x_{4} x_{6}+x_{1} x_{6} x_{7}+x_{2} x_{5} x_{7}+x_{2} x_{6} x_{7}+x_{3} x_{4} x_{6}+x_{3} x_{6} x_{7}+x_{4} x_{6} x_{7}+x_{5} x_{6} x_{7}\right)\right\}
\end{aligned}
$$


and

$$
C=\left(x_{1}+x_{2}+x_{3}+x_{4}+x_{5}\right)\left(x_{1}+x_{2}+x_{3}+x_{6}+x_{7}\right)-\left(x_{1}+x_{2}+x_{3}\right)^{2} .
$$

We introduce the Direct Computation Method (DCM), based on a combination of numerical integration and extrapolation on a sequence of integrals. DCM comprises the following three steps: (Step 1) $\varepsilon$ in Eq. (4.1) is set to a finite value determined by a (scaled) geometric sequence $\varepsilon=$ $\varepsilon_{l}=\varepsilon_{0} /\left(A_{c}\right)^{l}$ with $l=0,1, \cdots, \varepsilon_{0}$, and $0<1 / A_{c}<1$. (Step 2) Evaluate the multi-dimensional integral $I$ of Eq. (4.1) numerically. Because of the finite $\varepsilon_{l}$, we obtain a finite value for the integral corresponding to each $l$. Thus a sequence of $I\left(\varepsilon_{l}\right), l=0,1,2, \cdots$ is generated. (Step 3) Extrapolate the sequence $I\left(\varepsilon_{l}\right)$ to the limit as $\varepsilon_{l} \rightarrow 0$ to extract $I$ as $\lim _{\varepsilon \rightarrow 0} I(\varepsilon)$.

If $D$ does not vanish in the integration region, we can put $\varepsilon=0$ and we do not need an extrapolation process as $I=\left.I(\varepsilon)\right|_{\varepsilon=0}$.

We use Wynn's $\varepsilon$-algorithm [32,33] for the extrapolation, which works efficiently under fairly general conditions, even for very slowly convergent sequences. The $\varepsilon$ algorithm is applied to the sequence $I\left(\varepsilon_{l}\right), l=0,1, \cdots$ obtained by multi-dimensional integration.

We evaluate $I_{\text {non-planar }}$ given by Eq. (4.1). The kinematical variable $s$ is varied but $t$ is fixed at $t=-10000 \mathrm{GeV}^{2}$ throughout the computations. We introduce the dimensionless variable $f_{s}=$ $s / m^{2}$. For the mass parameters we set $m=m_{1}=m_{2}=m_{4}=m_{6}=50 \mathrm{GeV}, M=m_{3}=m_{5}=m_{7}=$ $90 \mathrm{GeV}$, and $p_{1}^{2}=p_{2}^{2}=p_{3}^{2}=p_{4}^{2}=m^{2}$.

Fig. 3 shows the results for $-20.0 \leq f_{s} \leq 20.0$. It is known that $I_{\text {non-planar }}$ has two cuts; one starts from the normal $s$-channel threshold, $s=4 m^{2}$, and the other from $s=-t-M^{2}-4 m M$ to $s=-\infty$. The latter corresponds to the $u$-channel threshold at $u=(M+2 m)^{2}$. The first and second cut correspond to $f_{s}=4.0$ and $f_{s}=-6.44$, respectively. In Fig. 3 we also show results for the imaginary part in the range $-100.0 \leq f_{s} \leq-20.0$. In this region the imaginary part is small but its contribution is not negligible when it is put in the dispersion integral;

$$
\mathfrak{R}(I(s))=\frac{1}{\pi}\left(\mathrm{P} \int_{-\infty}^{s_{0}^{\prime}} \frac{\mathfrak{I}\left(I\left(s^{\prime}\right)\right)}{s-s^{\prime}} d s^{\prime}+\mathrm{P} \int_{s_{0}}^{\infty} \frac{\mathfrak{I}\left(I\left(s^{\prime}\right)\right)}{s-s^{\prime}} d s^{\prime}\right),
$$

where $s_{0}=4 m^{2}$ and $s_{0}^{\prime}=-t-M^{2}-4 m M$ are the threshold in the $s$-channel and in the $u$-channel, respectively.

For the principal value integral computation, we used the trapezoidal rule, assuming that $\mathfrak{I}(I(s))=0$ far away from the origin, for $f_{s} \leq-100.0$ and $f_{s} \geq 50.0$. The results show good agreement with those by DCM. Thus the relation of Eq. (4.4) provides a consistency check of the numerical results produced by DCM.

\section{Summary}

We presented new results of GR @PPA 2.8, the event generator package for proton-proton and proton-antiproton collision, as a QCD application of GRACE. The matching method can be applied to single $W$ and $Z$ production processes and diboson $\left(W^{+} W^{-}, Z W\right.$ and $Z Z$ ) production processes.

We also developed the program package GRACE/SUSY-loop for the EW corrections and QCD corrections of the MSSM amplitudes at one-loop order. Then we calculated the radiative corrections to production processes and decay processes of SUSY particles, in the framework of MSSM, using GRACE/SUSY-loop. 


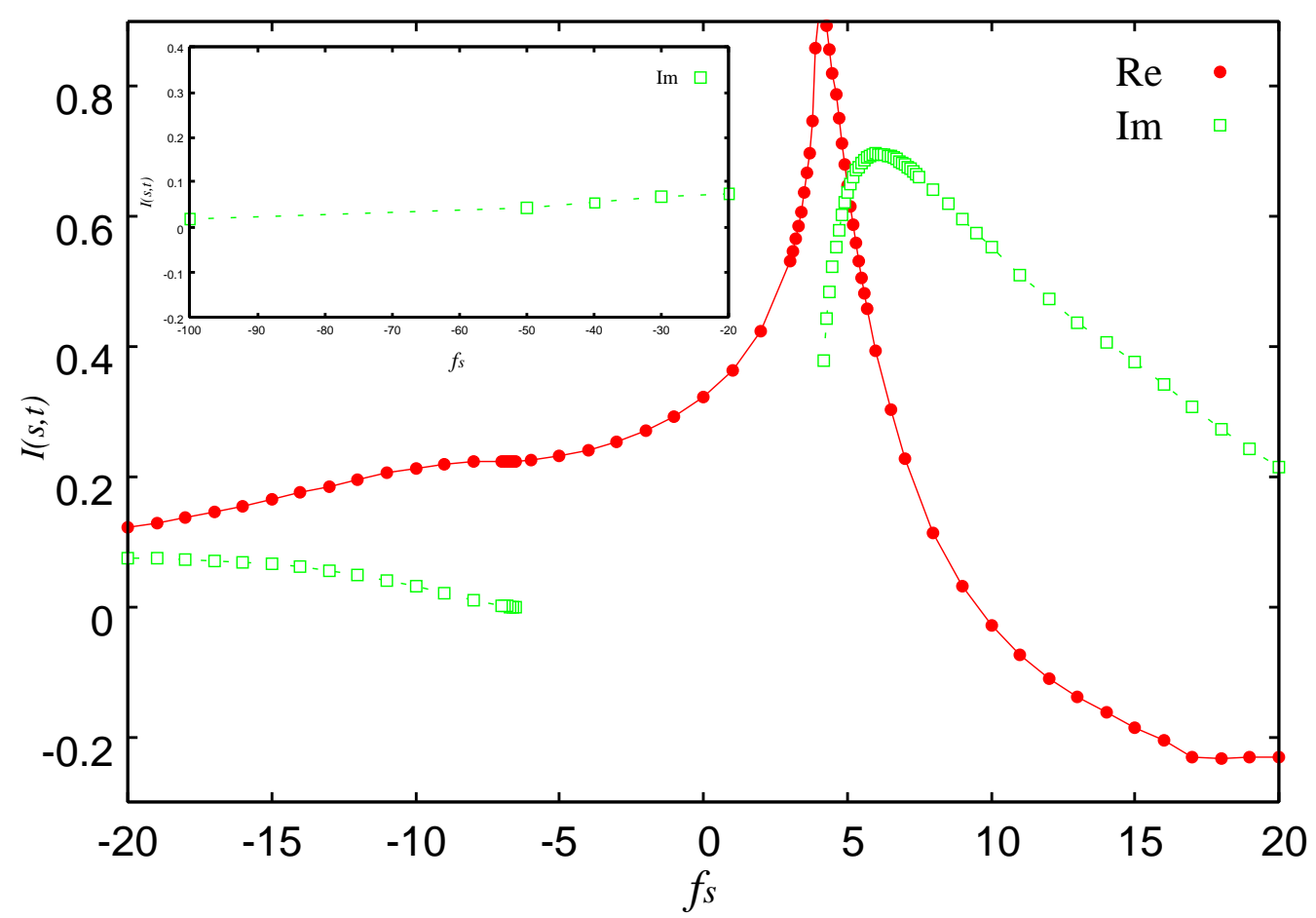

Figure 3: Numerical results of $\mathfrak{R}\left(I_{\text {non-planar }}\right)$ and $\mathfrak{I}\left(I_{\text {non-planar }}\right)$ for $-20.0 \leq f_{s} \leq 20.0$ and of $\mathfrak{I}\left(I_{\text {non-planar }}\right)$ for $-100.0 \leq f_{s} \leq-20.0$ in units of $10^{-12} \mathrm{GeV}^{-6}$ with $t=-10000.0 \mathrm{GeV}^{2}$. Plotted points are the real part (bullets) and the imaginary part (squares).

The calculation of the scalar integral of two-loop non-planar box diagrams involving massive particles, was shown as an example of the treatment of multi-loop integrals in the GRACE project. We introduced DCM for the evaluation of the loop integrals. The idea in DCM is that the $\varepsilon$ value in the propagators is treated numerically as a finite number. The usage of the dispersion relation is a powerful consistency check between the real and the imaginary part of the integral.

\section{Acknowledgements}

This work was supported in part by the Grant-in-Aid (No.20340063 and No.23540328) of JSPS, and by the CPIS program of Sokendai.

\section{References}

[1] S. Odaka and Y. Kurihara, [arXiv:1107.4467 ].

[2] Minami-Tateya Group, T. Ishikawa, et al., KEK-92-19 (1993).

[3] F. Yuasa, et al., Prog. Theor. Phys. Suppl. 138 (2000) 18; http://minami-home.kek.jp/ .

[4] S. Kawabata, Comput. Phys. Commun. 41 (1986) 127.

[5] S. Kawabata, Comp. Phys. Commun. 88 (1995) 309.

[6] S. Tsuno, et al., Comput. Phys. Commun. 151 (2003) 216.

[7] S. Tsuno, T. Kaneko, Y. Kurihara, S. Odaka, K. Kato, Comput. Phys. Commun. 175 (2006) 665. 
[8] S. Odaka, Mod. Phys. Lett. A25 (2010) 3047.

[9] A. A. Affolder, et al., Phys. Rev. Lett. 84 (2000) 845.

[10] B. Abbott, et al., Phys. Rev. D61 (2000) 032004.

[11] V. M. Abazov, et al., Phys. Rev. Lett. 100 (2008) 102002.

[12] J. Fujimoto, T. Ishikawa, Y. Kurihara, M. Jimbo, T. Kon and M. Kuroda, Phys. Rev. D75 (2007) 113002.

[13] J. Fujimoto, T. Ishikawa, M. Jimbo, T. Kon, Y. Kurihara, M. Kuroda and M. Tomita, Automatic calculation of SUSY particle production and decay with GRACE/SUSY-loop, Talk presented at TOOLS 2008.

[14] K. Iizuka, T. Ishikawa, Y. Kurihara, M. Kuroda, T. Kon, M. Jimbo and J. Fujimoto, 1 loop correction to sfermion decays with GRACE/SUSY-loop, Meeting abstracts of the Physical Society of Japan 63/2-1 (2008) 22.

[15] K. Iizuka, T. Kon, K. Kato, T. Ishikawa, Y. Kurihara, M. Jimbo and M. Kuroda, PoS RADCOR2009 068, [arXiv: hep-ph/1001.2800].

[16] M. Jimbo, K. Iizuka, T.Ishikawa, K. Kato, T. Kon, Y. Kurihara and M.kuroda, [arXiv: 1006.3491$]$.

[17] N. Baro and F. Boudjema, Phys. Rev. D80 (2009) 076010.

[18] T. Hahn, Nucl. Phys. Proc. Suppl. 89 (2000) 231; Comput. Phys. Commun. 140 (2001) 418.

[19] T. Fritzsche and W. Hollik, Nucl. Phys. Proc. Suppl. 135 (2004) 102.

[20] W. Öller, H. Eberl and W. Majerotto, Phys. Rev. D71 (2005) 115002.

[21] W. Killian, J. Reuter and T. Robens, Eur. Phys. J. C48 (2006) 389; AIP Conf. Proc. 903 (2007) 177.

[22] M. Drees, W. Hollik and Q. Xu, JHEP 0702 (2007) 032.

[23] W. Beenakker, R. Hopker and P. M. Zerwas, Phys. Lett. B378 (1996) 159.

[24] W. Beenakker, R. Hopker, T. Plehn and P. M. Zerwas, Z. Phys. C75 (1997) 349.

[25] J. Guasch, J. Solà and W. Hollik, Phys. Lett. B437 (1998) 88;

J. Guasch, W. Hollik and J. Solà, JHEP 10 (2002) 040; [arXiv: hep-ph/ 0307011$].$

[26] M. Jimbo, T. Inoue, T. Jujo, T. Kon, T. Ishikawa, Y. Kurihara, K. Kato and M. Kuroda, Talk presented at LCWS11.

[27] H. Eberl, W. Frischa, H. Hluchá, Nucl. Phys. Proc. Suppl. 205-206, (2010) 277;

H.Eberl, Fortschr. Phys. 58, (2010) 712.

[28] G. 'tHooft and M. Veltman, Nuclear Physics B153 (1979) 365.

[29] F. Yuasa, E. de Doncker, N. Hamaguchi, T. Ishikawa, K. Kato, Y. Kurihara. J. Fujimoto and Y. Shimizu, [arXiv:1112.0637].

[30] R. Piessens, E. de Doncker, C. W. Ubelhuber and D. K. Kahaner, Springer Series in Computational Mathematics. Springer-Verlag, 1983.

[31] S. Li, E. de Doncker, and K. Kaugars, Springer Lecture Notes in Computer Science (LNCS) 3514, (2005) 123.

[32] D. Shanks, J. Math. Phys. 34 (1955) 1.

[33] P. Wynn, Mathematical Tables Aids to Computing 10 (1956) 91. P. Wynn, SIAM J. Numer. Anal. 3 (1966) 91. 